\title{
Influence of Clay Addition on Physical Properties and Wettability of Peat-growing Media
}

\author{
Jean-Charles Michel ${ }^{1}$ \\ Agrocampus Ouest, EPHOR, 2 rue Le Nôtre, 49045 Angers, France
}

Additional index words. contact angle, WDPT, water repellency, water retention

\begin{abstract}
The role of clay incorporation on the physical properties and wettability of peatgrowing media was assessed from water retention curves and from contact angle and water drop penetration time measurements, respectively. Two peat substrates presenting different degrees of decomposition (weakly and highly decomposed Sphagnum peat) were used and mixed with clay in the form of powder with a peat:clay ratio of 90:10 (by vol.). Results indicated relatively little change in water retention resulting from clay incorporation in the peat-growing media tested. On the other hand, they showed a significant improvement of the wettability of both substrates in the driest conditions when peat-growing media presented a pronounced hydrophobic character, whereas the wettability of materials was not changed in the wettest conditions when they were largely hydrophilic. The influence of the degree of peat decomposition was also observed with somewhat higher wettability for the weakly decomposed peat:clay mixture than for the highly decomposed peat:clay mixture. Because the hydrophobic character of highly decomposed peat is more pronounced and appears at a higher water content than weakly decomposed peat, the effect of clay addition also appears at higher water contents for highly decomposed peat. Incorporating clay into peat-growing media should be considered for its ability to improve the wettability of growing media with a hydrophobic character (i.e., to improve the ability of the growing media to be rewetted) rather than only its ability to influence the water retention characteristics of the growing media.
\end{abstract}

Most growing media have a more or less pronounced hydrophobic character in relation to their moisture level (Michel et al., 2001). To remedy this, the addition of mineral materials or wetting agents has been proposed. However, the influence of these additives is often evaluated in terms of their effects on physical properties such as waterholding or air-filled capacity (Bunt, 1983; Martinez et al., 1997) or indirectly during the growing (Rivière et al., 1995) and rarely in terms of water repellency or hydrophobicity. Furthermore, the effects of theses additives on the relationship between water repellency and water content have never been investigated.

Water repellency (or wettability) of a material is defined by the ability of a liquid to spread on a solid surface and is then classically characterized by measuring the contact angle of a drop of liquid on the surface of the material (Letey et al., 1962) (Fig. 1). However, this method of measurement requires a smooth surface and, in the case of porous materials such as growing media, it thus requires the material to be compacted. As a consequence, this technique can only be used on dried materials (Jouany et al., 1992). However, the development of a

Received for publication 16 Mar. 2009. Accepted for publication 7 July 2009.

${ }^{1}$ To whom reprint requests should be addressed; e-mail Jean-Charles.Michel@agrocampus-ouest.fr. methodology already described by Michel et al. (2001) and based on the capillary rise principle now allows us to characterize the wettability of porous materials in relation to their water content. The change in contact angles from the hydrophilic to the hydrophobic character of peat samples during desiccation and the decrease in wettability related to the degree of peat decomposition have already been shown using this method (Michel et al., 2001). Both methods of contact angle measurements were coupled to the qualitative water drop penetration time (WDPT) test (McGhie and Posner, 1980), most often used to estimate water repellency of reconstituted soils (Dekker and Ritsema, 1994, 2000; Dekker et al., 2000; Doerr, 1998; Letey, 1969; McKissock et al., 2000).

On the basis of these studies, we questioned the efficiency of clay addition to improve the wettability of organic growing media. Clay and more or less decomposed peats were chosen as models because of the ability of peats to become hydrophobic during desiccation and because of the lack of studies concerning the direct effect of clay addition on water repellency in growing media compared with wetting agents. The objective was then to determine whether the incorporation of additives in growing media leads not only to a change in water retention characteristics resulting from solid phase reorganization, but to a change in wettability as well, improving the ability and speed of the growing medium to rewet.

\section{Materials and mixtures}

A weakly and a highly decomposed Sphagnum peat from Germany was chosen as a model to understand the influence of the degree of decomposition. These peat samples were selected for their similar particle size (fine fractions $0-4 \mathrm{~mm}$ ) and their very low ash content (less than $2 \%$ ). Clay was conditioned in the form of powder and has a varied mineralogical composition, including a prevalence of smectite $(65 \%)$, but also illite $(25 \%)$, kaolinite $(8 \%)$, and quartz $(2 \%)$. Mixtures were made in the wet state with a peat:clay ratio of 90:10 (by vol.).

\section{Physical properties}

Because the physical properties of growing media are largely influenced by how the materials are packed, the materials were prepared following the European standardized procedure NF EN 13041 (2000). Two large polyvinyl chloride (PVC) cylinders (diameter: $14 \mathrm{~cm}$; height: $14 \mathrm{~cm}$ ) were manually filled with substrates, slowly wetted (30 min) from the bottom, saturated for $24 \mathrm{~h}$, and then allowed to equilibrate to a water potential of $-3.16 \mathrm{kPa}$ during $48 \mathrm{~h}$. Cylinders were emptied, materials homogenized, and other small PVC cylinders (diameter: $10 \mathrm{~cm}$; height: $5 \mathrm{~cm} ; \mathrm{V}=393 \mathrm{~cm}^{3}$ ) were filled with each mixture without packing and slowly rewetted from the bottom for $24 \mathrm{~h}$. Four replications of each substrate were used in this experiment.

Water retention properties were determined using classical hydrostatic methods. For each of the four replicates, small cylinders were first placed on a tension table to drain the materials from $-1 \mathrm{kPa}$ to $-10 \mathrm{kPa}$. Suction levels between $-31.6 \mathrm{kPa}$ and -316 $\mathrm{kPa}$ were obtained by using a high-pressure chamber, whereas the suction level at -100 MPa was obtained from air-dried materials.

\section{Wettability measurements}

Contact angle measurements. The assessment of contact angles related to the water content required the use of complementary methods (capillary rise and droplet methods) as a result of their respective methodological limitations previously and widely described by Michel et al. (2001).

Contact angles on more or less wetted samples of peats and mixes were measured by the capillary rise method described by Michel et al. (2001). This method consisted of following the capillary rise on a column of materials with different liquids by using a Krüss Processor Tensiometer $\mathrm{K} 12^{\circledR}$ linked to a computer (Fig. 2). The speed of capillary rise, translated by the increase in weight of the sample, is measured in relation to time by the computer. The contact angle was determined from Washburn's (1921) equation, which defines the flow of a liquid through a capillary:

$$
\frac{l^{2}}{t}=\frac{\gamma_{L} \cdot r \cdot \cos \theta}{2 \cdot \eta}
$$

where $l$ represents the height of the wetting front $(\mathrm{cm}), t$ is the time $(\mathrm{s}), \eta, \gamma_{L}$ are the 
viscosity (mPas) and the surface tension of the liquid $\left(\mathrm{mJ} \cdot \mathrm{m}^{-2}\right)$, respectively, $r$ represents the radius of the capillary $(\mathrm{cm})$, and $\theta$ is the contact angle $\left({ }^{\circ}\right)$.

The powder that has been packed in a tube can be described as a bundle of capillaries with a mean radius of $\bar{r}$. The Washburn equation then becomes:

$$
\frac{l^{2}}{t}=\frac{\gamma_{\mathrm{L}}(\tau \bar{r}) \cos \theta}{2 \eta}
$$

where $\tau$ represents a constant to approximate the tortuosity of the capillaries.

By replacing the height of the wetting front $l$ by the increase in weight $m$ resulting from the penetration of the liquid through a bundle of $n$ capillaries, the Washburn equation becomes

$$
\frac{m^{2}}{t}=\frac{\pi^{2}(\tau \bar{r})^{5} n^{2} \rho^{2} \gamma_{L} \cos \theta}{2 \eta}
$$

and, more simply

$$
\frac{m^{2}}{t}=\frac{c \rho^{2} \gamma_{L} \cos \theta}{\eta}
$$

or even

$$
\cos \theta=\frac{m^{2}}{t} \frac{\eta}{\rho^{2} \gamma_{L} c}
$$

in which

$$
c=\frac{1}{2} \pi^{2}(\tau \bar{r})^{5} n^{2}
$$

where $m$ is the mass of the adsorbed liquid $(\mathrm{g}), \rho$ is the density of the liquid $\left(\mathrm{g} \cdot \mathrm{cm}^{-3}\right)$, and $n$ is the number of capillaries. The term $c$ $\left(\mathrm{cm}^{5}\right)$ corresponds to an empirical constant of the porosity and tortuosity of the capillaries, which depends on particle size and degree of packing.

This experiment was carried out on a small quantity of materials extracted from cylinders used for physical measurements and fixed at various water potentials $(-32$ $\mathrm{kPa},-100 \mathrm{kPa}$, and $-100 \mathrm{MPa}$ ). The parameter $c$ was initially assessed by using a liquid with a very low surface tension (i.e., hexane), which completely wets the sample $(\theta=0)$. The water/material contact angles could then be calculated and the wettability estimated knowing that the greater the contact angle, the more the hydrophilicity decreases or the hydrophobicity increases.

However, when the material is too hydrophobic (contact angles greater than $90^{\circ}$ ), there is no capillary rise, and the degree of hydrophobicity can then not be estimated using this technique. The droplet method, widely used in soil science and based on the direct contact angle measurement of a drop of water on a solid surface, is then used (Fig. 1). However, this requires a smooth surface and, in the case of our materials, measuring them on compacted and air-dried materials $(-100$ $\mathrm{MPa})$.

The 'water drop penetration time' test. Wettability was also monitored by using the WDPT test, which is commonly used by soil
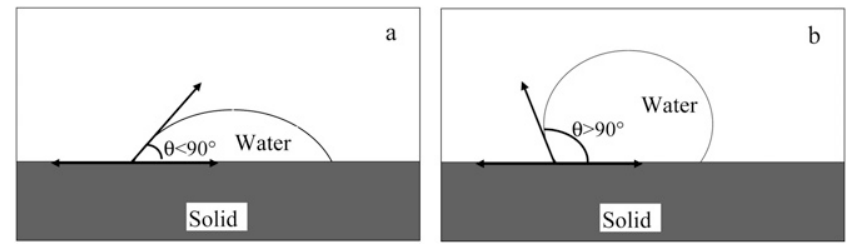

Fig. 1. Contact angle $(\theta)$ on a solid surface according to Young (1805). (A) hydrophilic material; (B) hydrophobic material.

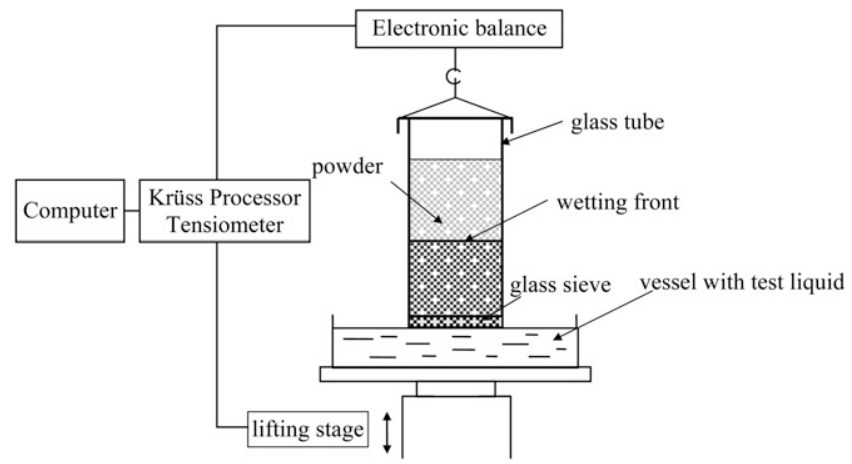

Fig. 2. Instrument for determining the contact angle on powders using the capillary rise method.

Table 1. Classification of soil/substrate water repellency related to the water drop penetration time. ${ }^{z}$

\begin{tabular}{llllll}
\hline $\begin{array}{l}\text { Water drop } \\
\text { penetration time }\end{array}$ & Less than $5 \mathrm{~s}$ & $5-60 \mathrm{~s}$ & $60-600 \mathrm{~s}$ & $600 \mathrm{~s}$ to $1 \mathrm{~h}$ & Greater than $1 \mathrm{~h}$ \\
\hline Surface state & Hydrophilic & $\begin{array}{c}\text { Weakly } \\
\text { hydrophobic }\end{array}$ & $\begin{array}{c}\text { Highly } \\
\text { hydrophobic }\end{array}$ & $\begin{array}{c}\text { Severely } \\
\text { hydrophobic }\end{array}$ & $\begin{array}{c}\text { Extremely } \\
\text { hydrophobic }\end{array}$ \\
\hline zDekker et al, 2000 & & & & &
\end{tabular}

Dekker et al., 2000.
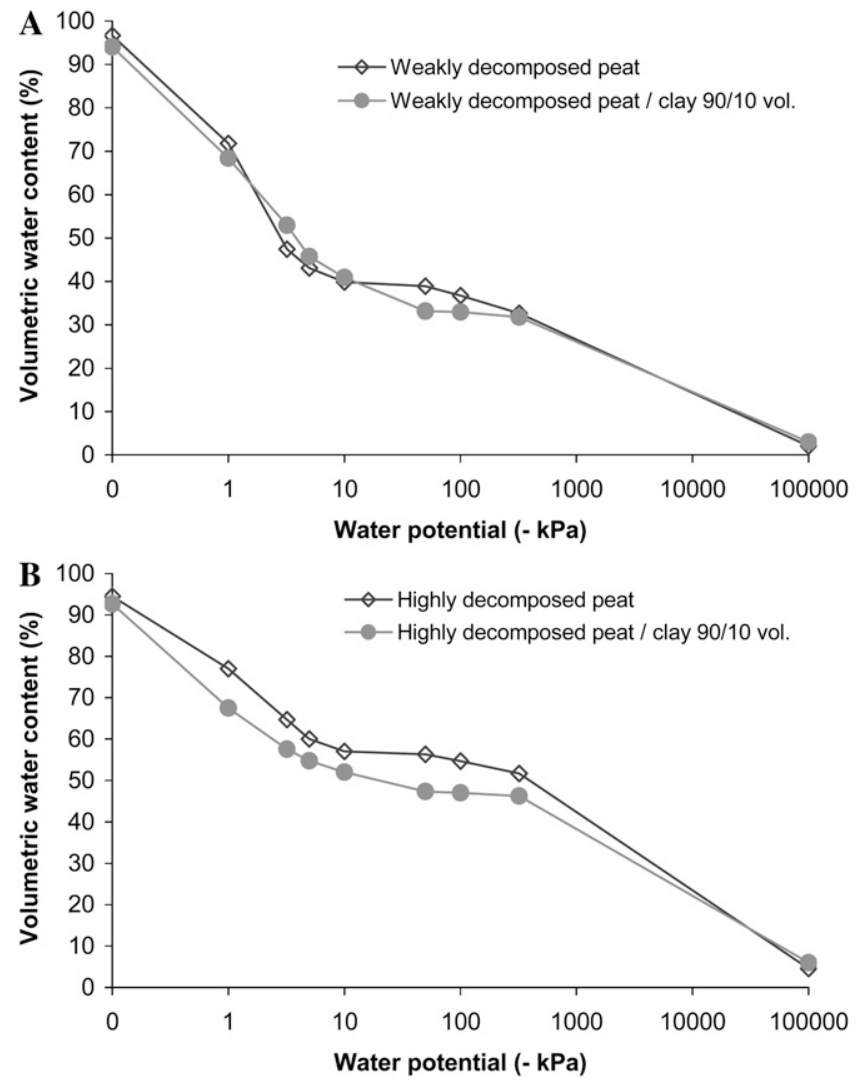

Fig. 3. Water retention curves of weakly and highly decomposed peats with or without $10 \%$ clay amendment. 
scientists, to compare results from WDPT tests with those from contact angle measurements.

This WDPT test was carried out on a small quantity of samples (peats and mixtures) at a water potential equal to $-100 \mathrm{kPa}$ by the centrifugation method (Michel et al., 2001) making it possible to obtain a material in the form of a galette with low rugosity (i.e., relatively smooth surface) and with a higher homogeneity in water content compared with cylinders used for physical properties. The materials were then progressively dried at 40 ${ }^{\circ} \mathrm{C}$ until completely dry, and WDPT measurements were carried out every $\approx 2$ hours. For each measurement, water content was previously measured; then 10 to 15 drops of distilled water $(10 \mu \mathrm{L}$ each) were deposited on the material using a syringe. Water repellency was then assessed using the classification presented by Dekker et al. (2000) (Table 1).

\section{Results and Discussion}

\section{Physical properties}

Water retention curves obtained on both of the pure peats are in agreement with results generally obtained on these types of materials (Fig. 3). Clay addition does not result in a major change in the water retention curves for both growing media, especially for weakly decomposed peat, whereas the water contents obtained are systematically slightly lower for highly decomposed peat after clay addition. This discrepancy (slight or no effect of clay addition) with the literature (Bunt, 1983; Martinez et al., 1997; Michel, 1998) can be explained by the fine particle size distribution of the peat samples used, which notably limits the decrease in water contents at high suction levels (from $-10 \mathrm{kPa}$ to $-316 \mathrm{kPa}$ ) for pure peats as compared with mixtures.

\section{Wettability measurements}

Contact angle measurements. Regardless of the growing media (with or without clay), a first analysis shows an increase in contact angles related to the degree of desiccation, in agreement with previous results (Michel et al., 2001) (Table 2). Desiccation of both peats led to a change from a hydrophilic character at higher water potentials $(-32 \mathrm{kPa}$ and $-100 \mathrm{kPa}$ ) to a hydrophobic character when the peat was air-dried (corresponding to a water potential equal to $-100 \mathrm{MPa}$ ). Variations according to the degree of decomposition were also observed for the pure peatgrowing media with an increase in contact angles for highly decomposed peat.

The peat:clay mixtures also showed an increase in contact angle during desiccation with the same trends in the increase of contact angles related to the degree of decomposition. However, significant differences could be observed in relation to clay addition. For weakly as well as for highly decomposed peats, clay addition did not reveal significant differences in contact angles at higher water potentials, but subsequently led to a progressive decrease in contact angles for drier materials, which is all the more important with the degree of desiccation. This leads to a

Table 2. Contact angles $\left({ }^{\circ}\right.$ ) at Sphagnum peat/water interface related to water potential (SDs are in brackets).

\begin{tabular}{lcccc}
\hline & \multicolumn{4}{c}{ Contact angles $\left(^{\circ}\right)$} \\
\cline { 2 - 5 } & $\begin{array}{c}\text { Weakly } \\
\text { decomposed } \\
\text { Weat }\end{array}$ & $\begin{array}{c}\text { Weakly } \\
\text { decomposed } \\
\text { peat/clay 90/10 vol. }\end{array}$ & $\begin{array}{c}\text { Highly } \\
\text { decomposed } \\
\text { peat }\end{array}$ & $\begin{array}{c}\text { Highly } \\
\text { decomposed } \\
\text { peat/clay 90/10 vol. }\end{array}$ \\
\hline$-32 \mathrm{kPa}$ & $71.4( \pm 1.9)$ & $70.8( \pm 1.6)$ & $76.2( \pm 1.1)$ & $75.7( \pm 2.1)$ \\
$-100 \mathrm{kPa}$ & $79.9( \pm 1.2)$ & $76.5( \pm 2.0)$ & $81.0( \pm 0.8)$ & $81.3( \pm 1.2)$ \\
$-100 \mathrm{MPa}$ & $107.3( \pm 2.0)^{\mathrm{z}}$ & $78.2( \pm 2.0)$ & $111.2( \pm 1.8)^{\mathrm{z}}$ & $88.5( \pm 0.2)$ \\
\hline
\end{tabular}

${ }^{\mathrm{z}}$ Data measured by the droplet method in contrast to the other data measured from the capillary rise method.
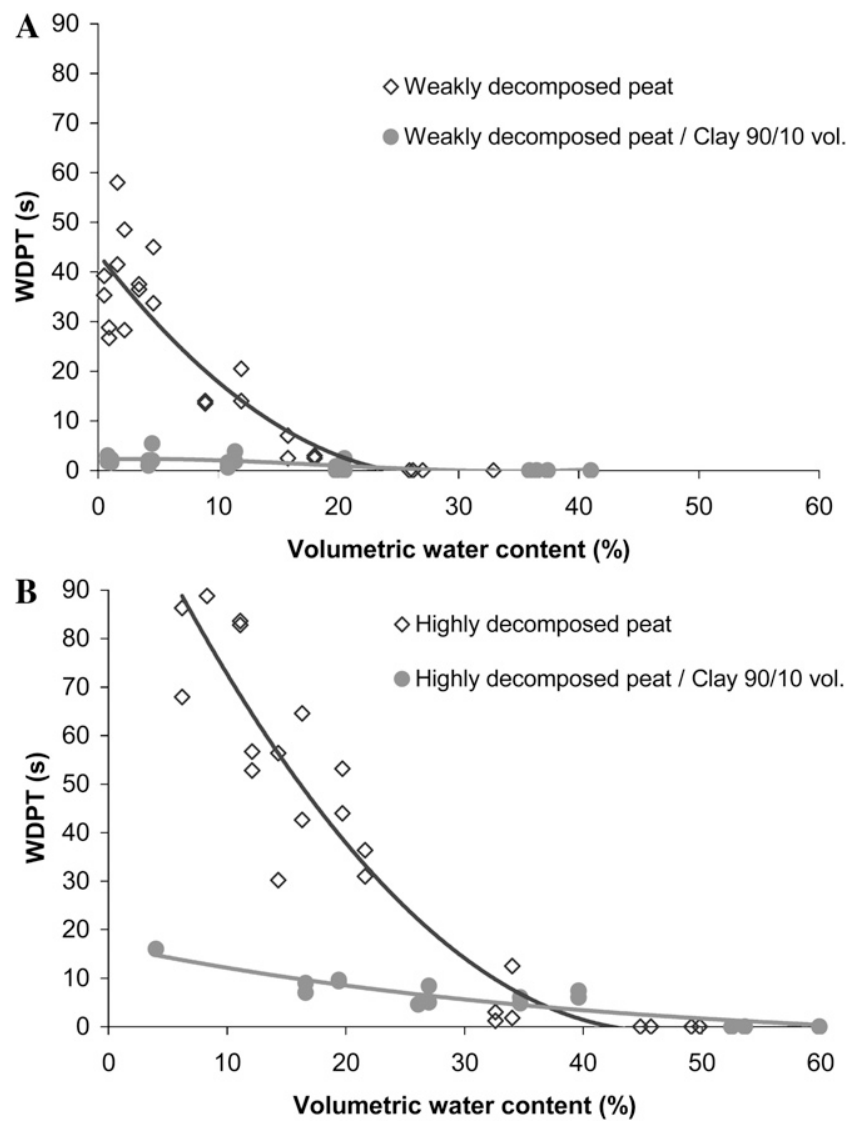

Fig. 4. Evolution of the water drop penetration time related to water content of weakly and highly decomposed peats with or without $10 \%$ clay amendment.

hydrophilic air-dried mixture in comparison with the hydrophobic pure peat. The results allowed us to conclude that the addition of clay seems to have a positive effect on the wettability of peat-growing media in the driest conditions, whereas no effect was observed for more wetted materials.

Water drop penetration time test. The analysis of the WDPT test obtained for weakly decomposed peat with or without clay did not show any difference for water contents higher than $20 \%$, because the materials were considered to be hydrophilic (WDPT less than $5 \mathrm{~s}$ ) according to the classification presented by Dekker et al. (2000) (Fig. 4). Differences appeared for water contents lower than $20 \%$, and the values obtained in the dry state suggested that weakly decomposed peat was a weakly hydrophobic material and that the weakly decomposed peat:clay mixture was a hydrophilic material.

For highly decomposed peat, a similar behavior was observed. However, differences in WDPT in the mixture appeared at higher water contents $(35 \%)$ than for white peat. This result is probably related to the lower wettability of highly decomposed peat compared with weakly decomposed peat. In the driest state, highly decomposed peat could be defined as highly hydrophobic material, whereas highly decomposed peat/clay was classified as weakly hydrophobic material.

If the increase in WDPT appeared at a higher water content for highly decomposed peat than for weakly decomposed peat, we could also observe that the WDPT is higher for highly decomposed peat than for weakly decomposed peat for the same water content provided that WDPT values are not equal to $0 \mathrm{~s}$. 
This observation was also verified after clay addition, but the differences between both mixtures were considerably lower. With or without clay, highly decomposed peat is therefore more hydrophobic than weakly decomposed peat, which is in agreement with previous results obtained on peats with different degrees of decomposition (Michel et al., 2001).

\section{Discussion and Conclusion}

The results obtained from contact angle measurements and WDPT tests are clearly related and thus make it possible to establish the positive influence of clay addition in peat on the wettability of materials. The improvement in wettability did not appear at high water contents when the materials were largely hydrophilic, but was demonstrated and then increased in relation to the degree of desiccation. Regardless of the growing medium, clay addition leads to an increase in wettability. However, the influence of the degree of peat decomposition was also observed after clay addition, with a smaller increase in wettability for a weakly decomposed peat:clay mixture than for a highly decomposed peat:clay mixture. Because the hydrophobic character of highly decomposed peat is more pronounced and appears at a higher water content than that of weakly decomposed peat, the effect of clay addition also appears at higher water contents for highly decomposed peat.

In general, clay incorporation in peat leads to a change in physical properties. However, this effect is more or less pronounced depending on the proportion of clay in the mixture and on the growing media tested and especially its particle size. In the case of our experiments, clay addition has little or no effect on the water retention properties of both of the growing media used and can be especially linked to the very fine fraction of peats tested, notably by limiting the decrease in water contents at high suction levels for pure peats in comparison with mixtures.

Although no significant change is revealed in terms of water retention with or without clay, wettability measurements, on the contrary, clearly showed large modifications in relatively dry conditions. In fact, clay addition does not affect wettability when the materials are wetted and hydrophilic, but contributes to its increase in the driest conditions when they present a more or less pronounced hydrophobic character. In summary, we can conclude that clay can be characterized by its ability to improve the wettability of growing media with a hydrophobic character, thus improving the ability and speed of the growing media to rewet.

\section{Literature Cited}

Bunt, A.C. 1983. Physical properties of mixtures of peats and minerals of different particle size and bulk density for potting substrates. Acta Hort. 150:143-160.

Dekker, L.W. and C.J. Ritsema. 1994. How water moves in a water repellent sandy soil. Water Resour. Res. 30:2507-2517.

Dekker, L.W. and C.J. Ritsema. 2000. Wetting patterns and moisture variability in water repellent dutch soils. J. Hydrol. 231-232:148164.

Dekker, L.W., C.J. Ritsema, and K. Oostindie. 2000. Wettability and wetting rate of Sphagnum peat and turf on dune sand effected by surfactant treatments, p. 566-574. In: Rochefort, L. and J.-Y. Daigle (eds.). Proc. 11th Int. Peat Cong. 6-12 August 2000, Quebec, Canada.

Doerr, S.H. 1998. On standardizing the 'WDPT' and the 'Molarity of an ethanol droplet' techniques to classify soil hydrophobicity: A case study using medium textured soils. Earth Surf. Process. Landf. 23:663-668.
Jouany, C., C. Chenu, and P. Chassin. 1992 Détermination de la mouillabilité des constituants du sol à partir des mesures d'angles de contact: Revue bibliographique. Science Sol 30:33-47.

Letey, J. 1969. Measurement of contact angle, water drop penetration time, and critical surface tension, p. 43-47. In: DeBano, L.F. and J. Letey (eds.). Proc. Symp. Water-repellent Soils. 6-10 May 1968, University of California, Riverside, CA.

Letey, J., J. Osborn, and R.E. Pelishek. 1962. Measurement of liquid-solid contact angles in soil and sands. Soil Sci. 93:149-153.

Martinez, F.X., N. Sepo, and J. Valero. 1997. Physical and physicochemical properties of peat-coir mixes and the effects of clay-material addition. Acta Hort. 450:39-46.

McGhie, D.A. and A.M. Posner. 1980. Water repellence of a heavy textured western Australian surface soil. Aust. J. Soil Res. 18:309-323.

McKissock, I., E.L. Walker, R.J. Gilkes, and D.J. Carter. 2000. The influence of clay type on reduction of water repellency by applied clays: A review of some West Australian work. J. Hydrol. 231-232:323-332.

Michel, J.C. 1998. Etude de la mouillabilité de matériaux organiques utilisés comme support de culture. Doctorat 3ème cycle, ENSA Rennes, France.

Michel, J.C., L.M. Rivière, and M.N. BellonFontaine. 2001. Measurement of the wettability of organic materials in relation to water content by capillary rise method. Eur. J. Soil Sci. 52: 459-467.

NF EN 13041. 2000. Amendements du sol et supports de culture-Détermination des propriétés physiques-Masse volumique apparente sèche, volume d'air, volume d'eau, valeur de rétraction et porosité totale. Association Française de Normalisation, Paris, France.

Rivière, L.M., N. Coulomb, and P. Morel. 1995. The use of clay in organic substrates for pot plants. Acta Hort. 401:97-105.

Washburn, E.W. 1921. The dynamics of capillary flow. Phys. Rev. 17:273-283.

Young, T. 1805. An essay on the cohesion of fluids. Trans. Royal Soc. London 95:65-87. 